\title{
Design of Automated Robotic System for Draping Prepreg Composite Fabrics
}

\author{
L.-P. Ellekilde $\uparrow$, J. Wilm $\ddagger$, O. W. Nielsen $\dagger$,

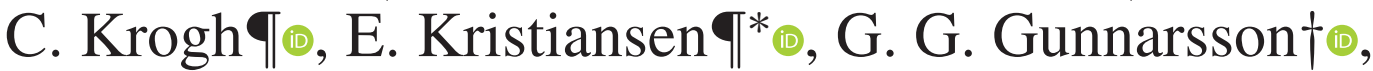 \\ T. S. Stenvang $\dagger$, J. Jakobsen $\uparrow$, M. Kristiansen $₫ \odot$, \\ J. A. Glud 9 , M. Hannemose $₫$, H. Aanæsł, J. de \\ Kruijk $\S$, I. Sveidahl||, A. Ikram\# and H. G. Petersen†๑
}

†University of Southern Denmark, Campusvej 55, 5230 Odense M, Denmark

E-mails:lpe@mmmi.sdu.dk,own@mmmi.sdu.dk, gunu@mmmi.sdu.dk,thss@mmmi.sdu.dk, hgp@mmmi.sdu.dk

$\ddagger$ Technical University of Denmark, Building 321, 2800 Lyngby, Denmark,

E-mails:jakw@dtu.dk,mohan@dtu.dk,aanes@dtu.dk

『Aalborg University, Fibigerstraede 16, 9220 Aalborg Ø, Denmark

E-mails:ck@m-tech.aau.dk,joj@m-tech.aau.dk,morten@m-tech.aau.dk,jag@m-tech.aau.dk

$\S$ Netherlands Aerospace Centre, Voorsterweg 31, 8316 PR Marknesse, The Netherlands

E-mail: joachim.de.kruijk@nlr.nl

||RoboTool A/S, Vestermarksvej 10, 6600 Vejen, Denmark

E-mail: is@ robotool.com

\#Terma Aerostructures A/S, Fabrikvej 1, 8500 Grenaa, Denmark

E-mail: aik@terma.com

(Accepted March 30, 2020. First published online: April 24, 2020)

\section{SUMMARY}

This paper presents a novel solution for precision draping of prepreg composite fabrics onto double curved molds. Our contributions relate to system design, including hardware and software components, and to system integration. On the hardware side, design and implementation of a drape tool with up to 120 suction cups positioned individually by linear actuators are described. On the software side, design and implementation of the software architecture are presented, along with necessary algorithms within sensor technologies and mathematical modeling. The essential system's components were verified individually, and the entire integrated system was successfully validated in the Proof-of-Concept experiments, performed on an experimental physical model of the system.

KEYWORDS: Industrial robotics; Composite manufacturing; System development; Automated draping process.

\section{Introduction}

Manual draping of woven composite fabrics onto one-of-a-kind molds is a time-consuming and labor-intensive process. When using fabrics, which are pre-impregnated with epoxy resin (prepreg material), the process also poses potential health risks to the operator. These factors hinder a more extensive application of composites in areas such as aerospace, wind turbines, automotive and shipbuilding. Hence, automation of the lay-up process could be highly beneficial. The FlexDraper

* Corresponding author. E-mail: ewa@m-tech.aau.dk 


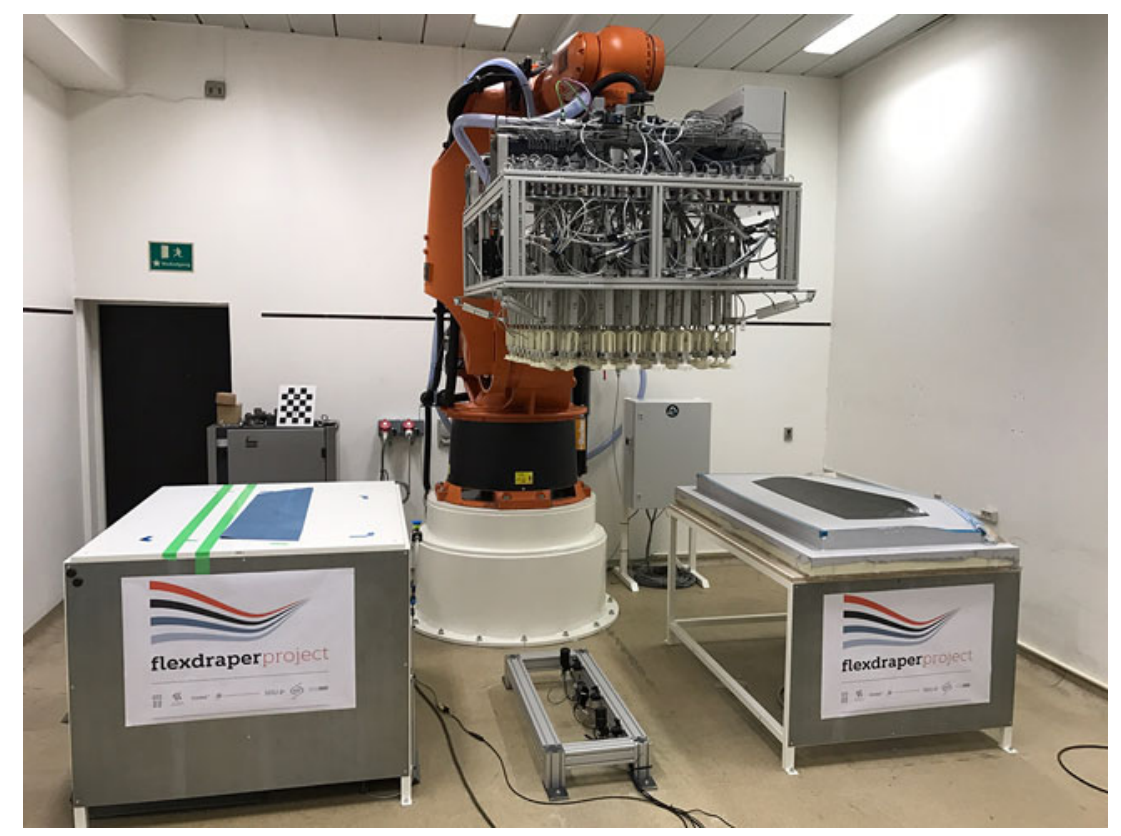

Fig. 1. Flexdraper, the developed experimental physical model of the system for draping the prepreg fabrics, which is comprised of an industrial robot, a drape tool with 80 suction cups on linear actuators, a picking table (on the left) and a mold table (on the right).

consortium was founded in 2013 to address this need and to develop a complete solution for automated draping of composite textiles. It consists of university and industrial partners with expertise in robotic systems, automation, vision, composite manufacturing and material modeling.

In this paper, we present a novel solution for high precision draping of prepreg composite fabrics onto one-of-a-kind double curved molds, using an automated robotic system, also refered to as a FlexDraper. Our contributions relate to the system design, including hardware and software components, and also to the system integration. The design of hardware components consists of mechanical design of the drape tool and design of a vision-based sensor system and relies on the kinematic and fabric modeling, verified in simulation. Special emphasis is put on design of software components for automatic planning of draping sequence and automatic planning of suction cup's trajectories for a given draping strategy. A strategy is defined here as a path initiated from a configuration where all suction cups are located above the mold (called the preshape) and end with all suction cups placed on the mold and the ply has been correctly draped. The draping strategy applied in this paper is inspired by manual draping in the wave-like pattern, where the drape starts by touching down at a well-chosen location from which the drape propagates over the lay-up surface with a wave-like movement. The aforementioned automatic planning requires an extensive modeling of fiber plies and a dynamic modeling of drape tool, which are both discussed in this article.

The drape task consists of picking pre-cut plies of prepreg fabric from a flat table surface and draping these onto the current stack of plies on a double curved mold. The pre-impregnated epoxy causes a certain stickiness, which makes it challenging to grip this material, and to complete the task without wrinkle formation. The developed FlexDraper system is shown in Fig. 1. The designed drape tool consists of up to $12 \times 10$ suction cups with individual linear actuators arranged in a rectangular grid, and it is moved by a six-axis industrial robot (Kuka KR360L2800). The integrated FlexDraper system for automated draping of prepreg composite fibers was successfully tested in the Proof-ofConcept experiments, performed on an experimental physical model of the system.

The paper is organized as follows: In the subsequent section, we discuss related approaches and existing systems for draping composite materials. Afterward, we present an overview of the proposed FlexDraper system. The succeeding section describes design and implementation of software architecture, followed by detailed insights into different system's components. Finally, the integrated system is validated in the Proof-of-Concept experiments, leading to a conclusion with an outlook toward future work. 


\section{State of the Art}

For automating the manufacturing of large composite structures such as aircraft hulls, the two main technologies are applied: Automated Fiber Placement and Automated Tape Laying. ${ }^{1}$ However, as documented in ref. [1], such systems are very expensive to acquire. Furthermore, their expected performance decreases significantly, when the parts become smaller. Therefore, the existing systems are not economically viable for smaller sized parts and in low volume production.

As stated in refs. [2,3], there have been earlier initiatives that attempted to find the automated and competitive solutions for smaller parts. The majority of them focus on dry fabrics without resin. Compared to the prepreg fabric, the processing and avoiding wrinkle formation is much easier with such dry fabric because the dry fabric is not tacky and it is more flexible. Within our scope, previous designs occur only in connection with R\&D environments.

Some examples of state-of-the-art robot end effectors for draping carbon fiber fabrics are described in refs. [2,4-8], which are based mainly on tools with multiple actuated gripper grids, capable of approximating the desired 3D shape. The articles refs. $[2,4,5,8]$ focus on dry fiber material, whereas the work presented in refs. $[6,7]$ is also discussing prepreg fabrics and has many similarities to ours. However, it only considers the tool development and does not describe the necessary planning, modeling and sensor systems described in this paper. An alternative to the aforementioned multigripper grids is presented in refs. $[9,10]$, which describes a cylindrical tool capable of draping dry fiber plies through a rolling motion. The work shows promising results for single curved parts, but it is not generalized to cope with double curved parts considered in FlexDraper.

One of the very few comparable automated pick and drape developments, which demonstrated several prototype parts, was successfully demonstrated by Netherlands Aerospace Center and Umeco in the COALESCE project and presented in refs. [11,12]. In COALESCE, a ply was picked up from a flat surface by a robot with an inflatable membrane end effector and the protective film was removed automatically. The material on the inflatable membrane was then slightly heated in order to improve both flexibility and tackiness. Afterward, the prepreg was draped onto a highly curved and complex mold. The system and process developed in COALESCE were found to be very cost-effective, but showed several drawbacks as well. Some of the plies needed to be cut into smaller pieces, which made it impossible to implement in the existing formal aerospace process qualification of the addressed part. Another disadvantage was that the robot was programmed manually, which did not allow for automatic adaptation to new parts.

To limit set-up and programming efforts, Bombardier and partners launched the Rapid Dry Carbon Fiber Lay-up program, ${ }^{13}$ which uses Computer-aided design (CAD) data to aid the automated movement coordination of their multi-gripper grid. This program goes one step further than COALESCE in terms of using available CAD data for automated movement coordination. However, it also only demonstrates the less challenging dry fiber material lay-up. Hence, even though there is a very high pressure within the market itself to lower production costs and maintain or increase quality for smaller prepreg parts, so far no complete solution has been demonstrated.

\section{Design of the FlexDraper System}

In this section, the designed FlexDraper system is presented, including an overview of its components. The FlexDraper system is built according to the flow chart shown in Fig. 2.

First, the suction cups are aligned to a co-planar array in the horizontal direction and then a sensorial measurement is performed to estimate the poses of suction cups. Knowing the positions of each suction cup, the ply is picked up from a given position on a table and the foil is removed. Since we have measured the positions of the suction cups relative to the robot, knowledge is available about the positions of all the suction cups relative to the ply at the moment when it is picked. Based on the $2 \mathrm{D}$ ply contour and the shape of the $3 \mathrm{D}$ mold, where the ply is to be placed, a method has been implemented that maps points on the 2D ply to a desired corresponding 3D points on the mold (see Section 6.1). Hence, knowing the pick locations of suction cups, the corresponding desired cup placements on the mold can be computed.

The Plan draping sequence component generates a sequence of suction cups' target poses. The first target pose of the suction cups in the sequence is the preshape and gives the shape of the ply immediately before the placement of the first suction cups onto the mold surface. The subsequent steps consist of selecting a first point of contact between the ply and the mold and from there 


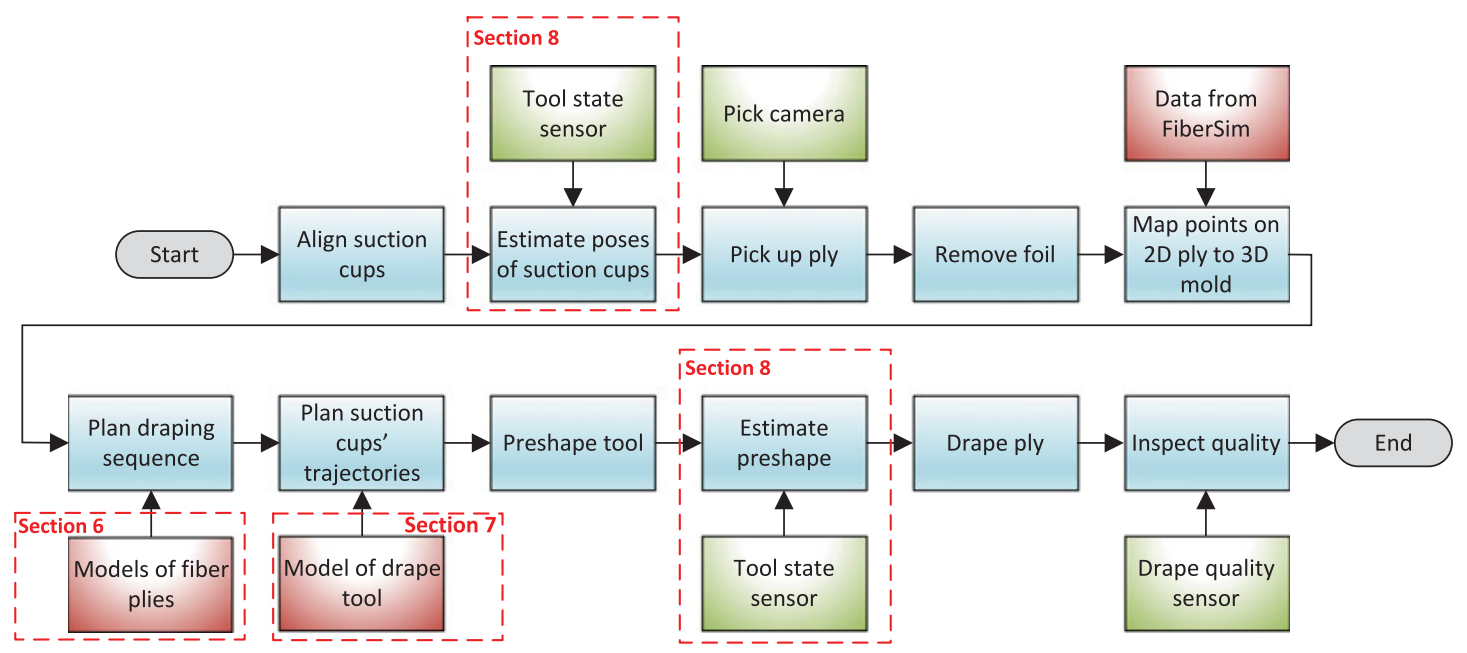

Fig. 2. Components in the FlexDraper system: the blue components represent the flow of actions, the green components are vision-based sensors and red components are data and computational models. The components encircled with the dashed, red line are explained in detail, in the referenced sections.

generating a wave-like pattern to perform the actual drape. In order to predict the draping outcome of this sequence, the system relies on the mathematical models of fiber plies presented in Section 6.2.

With a known sequence of suction cups' target poses, the Plan suction cup' trajectories component derives the trajectories of suction cups, using inverse kinematics and given the state of robot and all linear actuators. The drape tool mechanism is underactuated and with a non-trivial interrelation between the connected suction cups. Thus, a mathematical model (see Section 7) describing the dynamics of the tool has been developed and is used for simulating the tool behavior while computing the inverse kinematic solution, which minimizes the difference between the desired and actual suction cups' poses.

In order to account for inaccuracies in the mathematical models of the drape tool and the fiber plies, a custom vision-based sensor system is employed (see Section 8) and consists of Tool state sensor, Drape quality sensor and Pick camera. The Tool state sensor is employed to Estimate poses of suction cups and to Estimate preshape. The sensor data are providing the control variable for the actuators.

After the tool is preshaped and the preshape is estimated, the ply is moved to the mold and draped according to the planed sequence. Finally, the draped part is going through the Quality inspection system, which is relying on a Drape quality sensor and is intended for testing the draping quality with respect to specifications concerning location accuracy, avoidance of wrinkles and air intrusions.

\section{Hardware Design and Implementation of a Drape Tool and a Suction Cup}

In order to be able to lay-up plies that follow the very different contours of the lay-up mold accurately, it is necessary to have an end effector, which can be configured to the contours. For this purpose, we have developed an adaptive, actively manipulated gripper grid. Concerning the design of the gripper grid, several attributes and requirements had to be taken into account:

- The gripper must be able to handle plies of up to $1200 \times 1000 \mathrm{~mm}$ in size with a height variation of up to $150 \mathrm{~mm}$.

- Necessary lifting force of the individual suction cups must be sufficient.

- Distances between suction cups must be limited to be able to manipulate the material.

- Ability to comply with a wide range of lay-ups on double curved surfaces with small to medium curvature.

- The overall weight of the gripper must be within the payload limits of the robot.

- Electrical and mechanical complexity should be limited to improve robustness and reduce cost. 
(a)

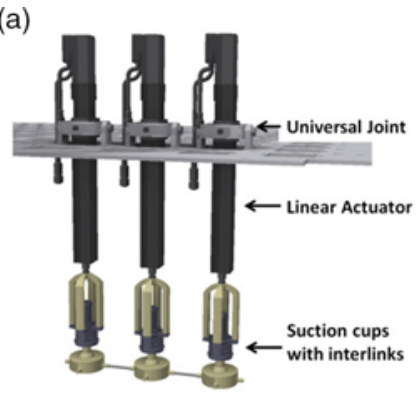

(b)

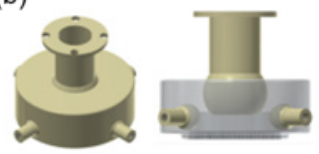

(c)

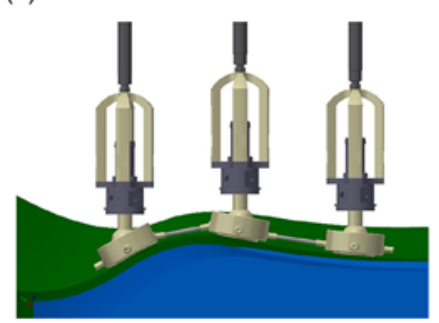

Fig. 3. Drape tool and suction cup design and functionality. (a) Part of tool showing the relative placement of the universal joints, actuators and suction cups. (b) Close-up of suction cups with ball joints for connecting with the actuator and interlink structure. (c) Illustration of interlink structure when placed at different heights.

Throughout the experimentation, we found out that to enable the preshape with medium curvature and details, the distances between suction cups need to be rather small. This conclusion also supports the criterion for necessary lifting force and limited free-hanging material. On the current FlexDraper tool, a grid spacing of approximately $110 \mathrm{~mm}$ between adjacent suction cups has been selected. The chosen spacing distance imposes the requirement of up to $12 \times 10=120$ grippers to handle the specified part dimensions. Considering the weight and complexity issue, the number of actuators should be limited. We have therefore chosen to use only one linear actuator per suction cup for up/down movements and employ passive joints and interlinks for allowing the cups to adjust the horizontal position and comply the orientation to the mold. Figure 3 a highlights a part of the tool with three suction cups and interlinks.

We use SCHMALZ Composite Grippers (SCGs), which create suction by means of the Venturi effect. The grippers are equipped with a custom-designed suction cup housing (see Fig. 3b) with integral ball joints, which can turn up to \pm 40 degrees. A set of so-called interlinks between the suction cups have been attached to force the position and orientation of suction cups and naturally adjust to a surface of changing height (see Fig. 3c).

The suction cups are positioned by electric linear actuators with spindle drive (FESTO EPCO-16150-3P-ST-E). The actuators can be moved individually and can use position, velocity and force for feedback control. The stroke length of the FESTO actuators (Z-direction) is $150 \mathrm{~mm}$. The gripper sub-assemblies of linear actuators and SCGs with custom suction cup housings are mounted equidistantly on a base plate. To passively account for the changes in horizontal distance of the suction cups from a $2 \mathrm{D}$ flat surface to a 3D-shaped contour, we mount the actuators on universal joints allowing them to rotate and thereby satisfy the constraints of interlink structure. Even with this drape tool design consisting of only one actuator per suction cup, the total weight of the whole assembly due to the actuators, control boxes, power supplies, pneumatic components, wiring and tubing is adding up to approximately $250 \mathrm{~kg}$.

Finally, it should be mentioned that in order to be able to handle different ply shapes, suction will only be applied to the relevant areas of the multi gripper grid. Not only because of air/energy consumption but also due to the fact that the inactive grippers also cause loss of suction when they are on the same local air supply circuit as active grippers. Additionally, the suction control prevents the potential problem of laying down plies not covering the complete area of the previous ply, as inactive grippers with suction may get close to the previous yet not fully attached ply. 


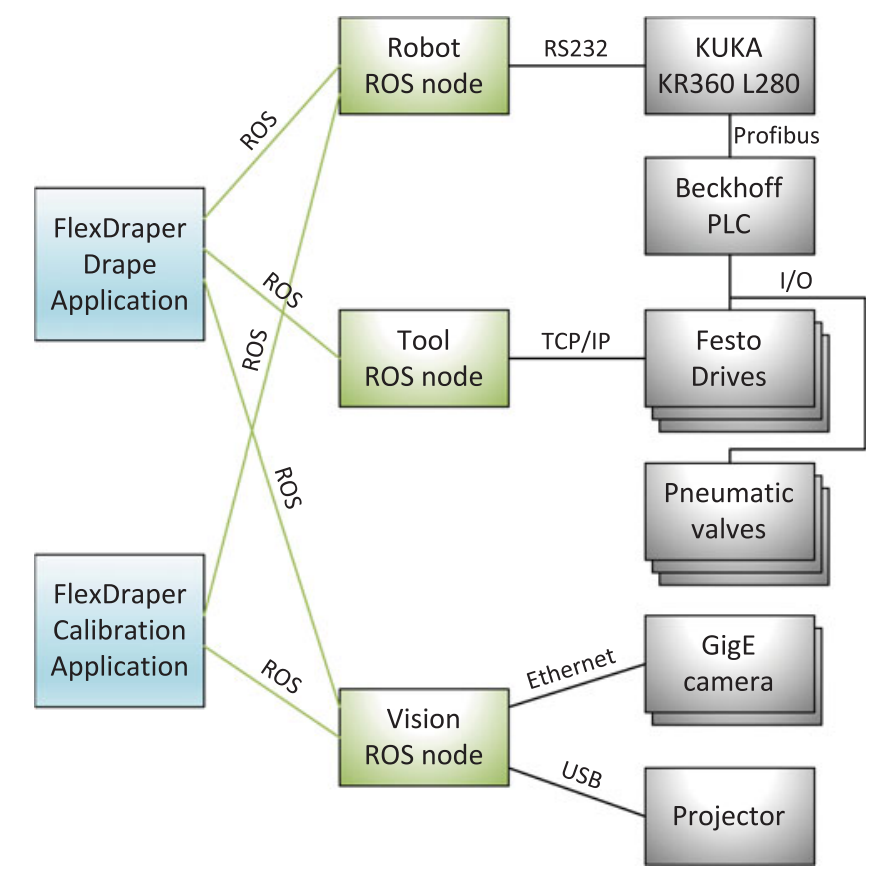

Fig. 4. System architecture showing the application components (blue), ROS components implementing functionality blocks and hardware abstractions (green) and hardware entities (grey).

\section{Design and Implementation of Software Architecture for a FlexDraper System}

This section describes the software architecture for the FlexDraper system. In order to bind its components together, the system uses $\operatorname{ROS}^{14}$ as a middleware for enabling functionality to be distributed across multiple processes and computers. At the same time, ROS also provides a natural decoupling of components enabling project partners to work in parallel and update functionality as long as the components comply to the agreed ROS service interfaces.

The main system architecture is shown in Fig. 4 with the different application components shown to the left. These currently includes: (1) FlexDraper Drape Application, which implements the drape planning and execution strategy and (2) Calibration Application used for calibrating the robot to the sensors and to the mold.

The second column in Fig. 4 represents nodes responsible for a specific functionality, such as communication with the robot and the tool. The ROS interfaces for these components are designed to be hardware independent, which allows for future upgrades of hardware without changing the application components.

The right column in Fig. 4 represents the hardware entities and not ROS nodes. A Kuka KR360L280 robot is controlled through an RS232 serial interface. The communication follows a client-server pattern with the robot being the client polling the server for tasks. The robot has the capability of buffering up to $N$ tasks enabling it to blend smoothly between motions. Besides pointto-point and linear motions, the current interface also supports setting and reading $\mathrm{I} / \mathrm{O}$, reading the robot configuration and wait statements.

The main communication with the tool is done through Transmission Control Protocol/Internet Protocol (TCP/IP), where each of the controllers for the FESTO actuators has their own IP address. Using the TCP/IP connection, the controllers are configured individually from the ROS node with desired goals and velocity profiles. To ensure a synchronized start of the actuators, they are set up to start based on a digital I/O signal. The valves used for controling suction of the tool are likewise controlled through digital I/O. All mentioned I/O signals are connected to a Beckhoff PLC build into the tool and controlled from the robot using ProfiBus. This solution provides a great flexibility, as the Beckhoff PLC can easily be extended, and it does not require placement of additional wires along the robot in case of a need for more I/O signals. 
Sensor systems and analysis are performed on a separate computer, which serves ROS nodes, so that the measurement updates can be requested and the results can be returned to the FlexDraper Drape Application.

\section{Models of Fiber Plies}

The first purpose of modeling the fiber plies is to calculate the optimal, final 3D location $r_{m o l d}(u, v)$, for any point $(u, v)$ on the ply. The second purpose of modeling the fiber plies is to create an engine for planning draping strategies and for predicting the result of trial draping strategies. Since many trial strategies may be required before obtaining the correctly draped ply, it is important to have fast computable, but not necessarily highly accurate models for the initial planning. On the other hand, at or near the final solution, the models must be accurate, potentially at the expense of a longer computation time. Therefore, the studies are conducted on both less accurate, but fast approximate models and highly accurate, but computationally expensive models.

\subsection{Estimating the transformation $r_{\text {mold }}(u, v)$}

An initial estimation of the transformation of a point $(u, v)$ on the flat ply on the picking table to a corresponding point on the curved mold geometry $r_{\text {mold }}(u, v)$ is done by a surface mapping algorithm. The algorithm is based on work by ref. [15] and extended with ply boundary constraints. It serves to compute the position and orientation of the suction cups on the mold based on the contact points between suction cup and ply during the pick up. The algorithm is based on a minimization of the energy density in the fibers of the ply material. Therefore, the solution seeks to minimize fiber deformations. Prior to initiating the minimization routine, a starting point is singled out and then a fiber together with its orthogonal at that point are mapped to the mold. A discretization size is chosen, and the energy minimization of the domain described by $r_{\text {mold }}(u, v)$ is executed.

\subsection{Computationally fast models}

Computationally fast models of the fiber ply deformations during draping are required for efficient execution of the initially broad search space of the drape planning. The typical approach for fast models of fabrics and other flexible materials is a damped mass spring model (see for example ${ }^{16}$ ). However, for the prepreg material used in this project, the stiffness in the fiber direction leads to very stiff springs and hence requires a very small time step, leading to slow computations. Rather than using the mass spring model, a static geometric model has been developed. This model takes advantage of the very high arc length stiffness in the fiber directions and computes only the equilibrium solution and not the internal modes, which are due to, for example, accelerations of the drape tool.

The following assumptions have been made in our model.

- Gravity can be neglected compared to material forces.

- Vibrational modes can be neglected, so only the equilibrium solution is needed.

- The material is first-order continuous at all points.

- The arc length in fiber directions between adjacent suction cups is preserved.

- There is no sliding of material relative to the suction cups.

With these assumptions, the ply can be modeled by a fiber length preserving polynomial interpolation. A combination of third- and fourth-order polynomials has been used.

$$
\mathbf{r}^{*}(s)=\mathbf{a}_{\mathbf{3}}^{*} s^{3}+\mathbf{a}_{\mathbf{2}}^{*} s^{2}+\mathbf{a}_{\mathbf{1}}^{*} s+\mathbf{a}_{\mathbf{0}}^{*}
$$

The $\mathbf{a}^{*}$ parameters can be found using boundary conditions. By adding a length constraint a fourthorder polynomial can be described as:

$$
\mathbf{r}(s)=\mathbf{r}^{*}(s)+a_{4}^{*} \mathbf{d} s^{2}(L-s)^{2} .
$$

$L$ denotes the length of a fiber between two constrained points and $\mathbf{d}$ is defined as:

$$
\mathbf{d}=\mathbf{r}\left(\frac{L}{2}\right)-\frac{1}{2}(\mathbf{r}(0)+\mathbf{r}(L))
$$


In order to compute $a_{4}^{*}$, it is assumed that $L$ is known and that the arc length in parametric form is given by:

$$
L \equiv \int_{0}^{L} \sqrt{\mathbf{r}^{\prime} \cdot \mathbf{r}^{\prime}} d s .
$$

Using Eqs. (1)-(4) for selected fibers connecting suction cups in both directions expands a grid. The enclosed areas can then be interpolated by a location-dependent weighed average, resulting in a function of the form. $r(u, v)=\{x(u, v) ; y(u, v) ; z(u, v)\}$, where $u, v$ describe the $2 D$ location on the ply and $r(u, v)$ describes the corresponding $3 D$ location as a function of the position and orientation of the four nearest suction cups. For a more detailed derivation refer to ref. [17]. The advantage of this model is that it fits the experimental data well and that it is computational cheap. For an $10 \times 8$ gripper grid and with non-optimized MATLAB code, the model is using approximately $0.6 \mathrm{~s}$ on a conventional PC. The fact of working with an equilibrium solution makes the found parameters path invariant. It reduces the search space drastically, as it allows for the draping strategies to be tested by simulating subparts of the strategy separately. This contributes to further reduction of computational time.

\subsection{Advanced FEM type models}

In order to simulate the outcome of a finalized draping sequence and to confirm that the planned suction cups' trajectories that were learned and programmed using the computationally fast model are acceptable, a highly accurate model is developed. The accurate model considers the peculiar mechanical responses of the prepreg. Due to the arrangement of the fibers in the ply, the fibers can rotate in the weave, i.e. shear, such that the initial $90^{\circ}$ fiber angles change. As a consequence, the material is highly anisotropic, i.e. the stiffness in the fiber direction is higher than in diagonal directions by several orders of magnitude. In addition, the stiffness is non-linear in the deformation (strain) as well as dependent on the rate of deformation due to the presence of the uncured resin (viscoelasticity). At large straining, permanent deformations, such as plasticity can come into effect. The tackiness of the ply affects the frictional properties in the interfaces between the ply and the suction cups as well as in the interface between the ply and the mold. This in turn affects whether the ply slides or deforms when being manipulated with the suction cups. These transient aspects of the draping must therefore also be considered in a highly accurate model.

A closed form solution that can represent the mechanical response for arbitrary ply geometries is not available, and thus the Finite Element (FE) method is deployed. Numerous accounts of successful application of FE simulations of woven reinforcement drapings are found in the literature. ${ }^{18}$ Here, a so-called continuous model is applied, where homogenization theory and shell elements are used for modeling the otherwise non-homogeneous nature of the plies. Our material model is based on the measured material response from standardized tests. The in-plane ply response can be measured using a universal testing machine, where the force required to elongate a ply sample is recorded. For the fiber direction response, the fibers must be aligned with the direction of loading. To measure the shear, i.e. change in fiber angles, the sample must be oriented $45^{\circ}$ to the loading direction as seen in Fig. 5a. This $45^{\circ}$-test is known as the bias-extension test. ${ }^{19}$ By means of kinematics, the sample elongation can be converted to the change in shear angle and the recorded load can be converted to the shear load in the ply. The results from a bias-extension test on the prepreg material for three different displacement rates are presented in Fig. 5 b.

Relevant output of the FE model includes: undesired formation of wrinkles, resulting local fiber angles, amount of shear and reaction forces at the interfaces that can be used for identifying sliding effects. Although the FE model requires a substantial number of inputs and the solution time can be in the order of hours, it is a powerful tool for offline studies and for verification of draping strategies. An example of the results from the FE model is shown in Fig. 6. Details on the material characterization, together with the FE modeling and the results can be found in ref. [20].

\section{Model of Drape Tool}

Initially, there conducted a study on the resulting degrees of freedom related to an underactuated tool. Consider here an $M \times N$ array of suction cups and hence $M \times N$ actuators. As each suction cup has 6 degrees of freedom, there would be $6 M N$ degrees of freedom if no constraints were present. With 
(a)

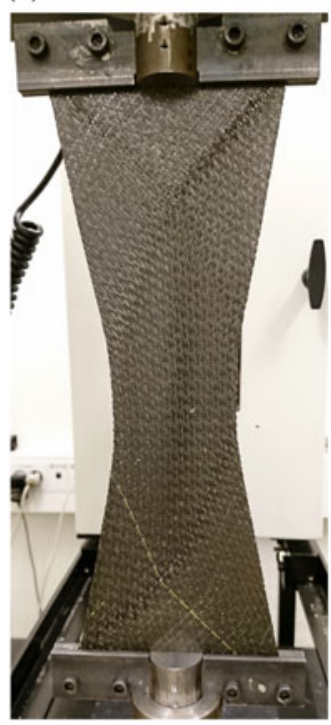

(b)

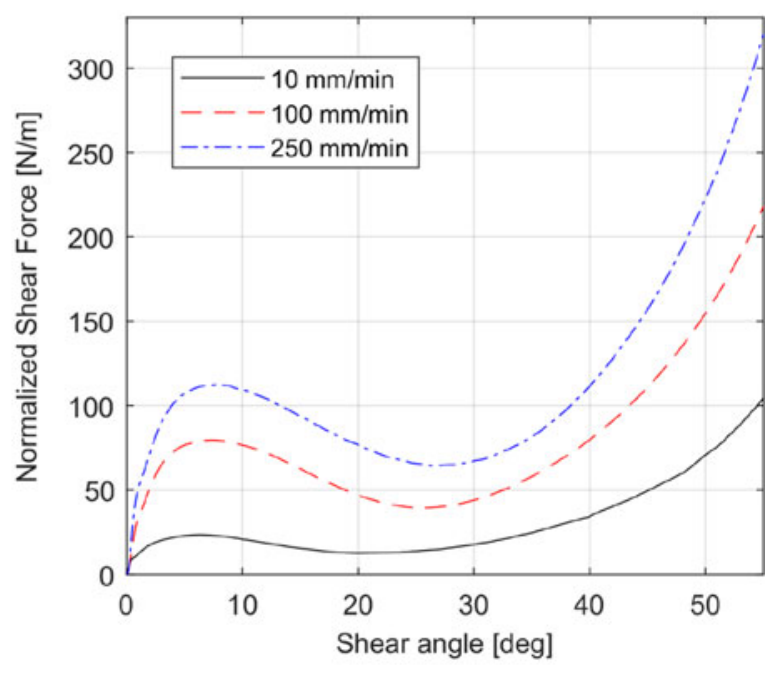

Fig. 5. The bias-extension test. (a) Test set-up with $120 \times 270 \mathrm{~mm}$ sample. (b) Averaged shear force vs shear angle results. Three samples were tested for each rate.
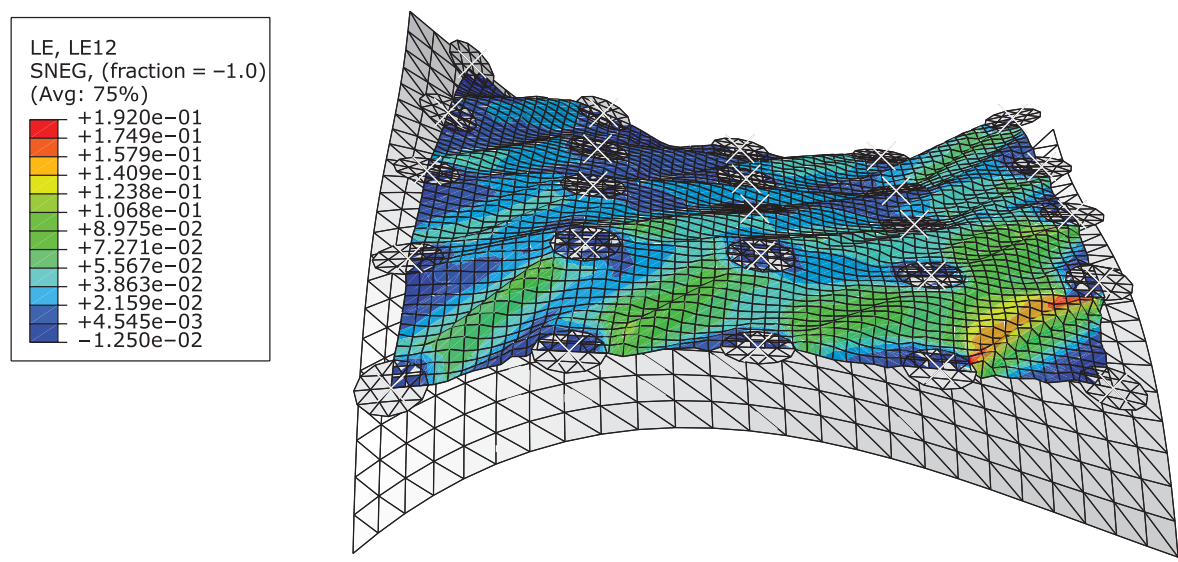

Fig. 6. A FE model of a $5 \times 5$ grid of suction cups in a free-hanging configuration. The colors indicate amount of shear.

the actuators at fixed configurations, there are $M N$ actuator constraints and $M(N-1)+N(M-1)$ interlink constraints. This results with an underactuated system with $3 M N+M+N$ internal degrees of freedom when the actuators are locked.

The underactuated drape tool has all the advantages previously listed, but the disadvantage is that the underactuation increases the difficulty of drape tool modeling. However, our experiments have indicated that at least there is a high degree of repeatability of the configurations when the plies are attached because the forces between the suction cups and the material determine the configuration.

This indicates that it is possible to model these forces correctly and thus predict the suction cup positions. To exploit that, a model is developed, which takes these forces as input and computes the resulting suction cup configuration. Additionally, a so-called Tool state sensor, described in the Section 8 , is measuring the actual location of the suction cups, which are first used for performing an iterative fine tuning of the model and afterward for validating the accuracy of the final model.

Each of the $M N$ suction cup assemblies, shown in Fig. 3, is modeled as three rigid bodies. These bodies are: the actuator housing, the actuator rod and the suction cup. The inertia is defined relative to the rigid bodies and the velocities as spatial twists. ${ }^{21}$ The generalized coordinates, $q_{i}$, for assembly 
$i$ are: $q_{i}=\left[\theta_{y, i}, \theta_{x, i}, \eta_{i}, \phi_{z, i}, \phi_{y, i}, \phi_{x, i}\right]^{T}$. The $\theta$ 's are denoting the angles of the universal joint at the mounting point, first rotating about the $y$-axis and then the resulting $x$ axis. $\eta$ is the extension of the actuator, and the $\phi$ 's are the $z-y-x$ Euler angles of the suction cup. The total state vector $q$ is made by stacking all the $q_{i}$ vectors. The total kinetic energy of the whole tool using generalized coordinates is then:

$$
T=\frac{1}{2} \sum_{i=1}^{M N} \sum_{j=1}^{3}\left[m_{j}\left\|\dot{\mathbf{p}}_{j}\left(q_{i}\right)\right\|^{2}+\omega_{j}^{T}\left(q_{i}\right) \cdot \mathbf{I}_{j} \cdot \omega_{j}\left(q_{i}\right)\right]=\frac{1}{2} \dot{q}^{T} \cdot \mathbf{M}(q) \cdot \dot{q},
$$

where $m_{j}$ is the mass of the jth body, $\dot{\mathbf{p}}_{j}\left(q_{i}\right)$ is the time derivative of the center of mass of the body, $\omega_{j}\left(q_{i}\right)$ is the angular velocity and $\mathbf{I}_{j}$ is the inertia matrix. The matrix $\mathbf{M}(q)$ is the generalized inertia matrix.

The interlink constraints are modeled as point-to-point constraints between assemblies. The rotation point of the interlink on each suction cup is defined as $\mathbf{r}_{\alpha}$ and $\mathbf{s}_{\alpha}$ for each interlink $\alpha$. Then, the interlink constraint is expressed as:

$$
f_{\alpha}(q)=\frac{1}{2}\left(\left\|\mathbf{r}_{\alpha}(q)-\mathbf{s}_{\alpha}(q)\right\|^{2}-d^{2}\right)=0, \quad \alpha=1, \ldots, M(N-1)+N(M-1),
$$

where $d$ denotes the length of the interlink.

The movement profiles of the actuators are known and are modeled as time-dependent constraints on the $\eta$ coordinates:

$$
g_{\beta}(t, \eta)=\eta_{\beta}-c_{\beta}(t)=0, \quad \beta=1, \ldots, M N,
$$

where $c_{\beta}$ is the movement of the $\beta$ 'th actuator.

The potential energy is denoted by $V(q)$, and the damping forces in the tool, denoted by $\mathcal{F}(\dot{q})$, is derived by defining a Rayleigh dissipation function. ${ }^{22}$

Using constrained Lagrangian mechanics, ${ }^{23}$ the equations of motion become:

$$
\begin{aligned}
\frac{d}{d t} \partial_{\dot{q}} T(q, \dot{q})-\partial_{q} T(q, \dot{q})+\partial_{q} V(q)+\partial_{\dot{q}} \mathcal{F}(\dot{q}) & =\sum_{\alpha} \lambda_{\alpha} \partial_{q} f_{\alpha}(q)+\sum_{\beta} \mu_{\beta} \partial_{q} g_{\beta}(t, q) \\
\partial_{q} f_{\alpha}(q) \cdot \ddot{q}+\dot{q}^{T} \cdot \partial_{q q} f_{\alpha}(q) \cdot \dot{q} & =0 \\
\ddot{\eta}_{\beta}-\ddot{c}_{\beta}(t) & =0
\end{aligned}
$$

In Eq. (8), the variables $\lambda$ and $\mu$ are Lagrange multipliers for the interlink constraints and the actuator constraints, respectively. The operator $\partial$ denotes the partial derivative with respect to the variable in the subscript. Equations (9) and (10) are acceleration constraints due to the interlinks and actuator movement. The acceleration constraints introduce drift in the position constraints during integration, which is mitigated using mass orthogonal projection ${ }^{24}$ at every timestep.

\section{Sensor System}

Automated optical (vision) measurements are needed for performing several sub-tasks in FlexDraper. More specifically, the sensor system shown in Fig. 7 was designed. It consists of:

- Pick camera. This is an industrial camera mounted above the pick table. The contour of a ply is found using image processing algorithms.

- Tool state sensor. This is a structured light vision system positioned between pick and drape tables. Details of the sensor are given below.

- Drape quality sensor. This is an additional higher resolution structured light vision system. This sensor will be mounted above the drape table to measure the ply surface after a drape. The allowable tolerance for ply placement is $+/-2.5 \mathrm{~mm}$ on the outer contour and $+/-3$ degree for the fiber angle at a specified point. Hence, the measurement accuracy must be below these tolerances. The drape quality sensor has not been installed yet as the draping quality is still manually inspected. 


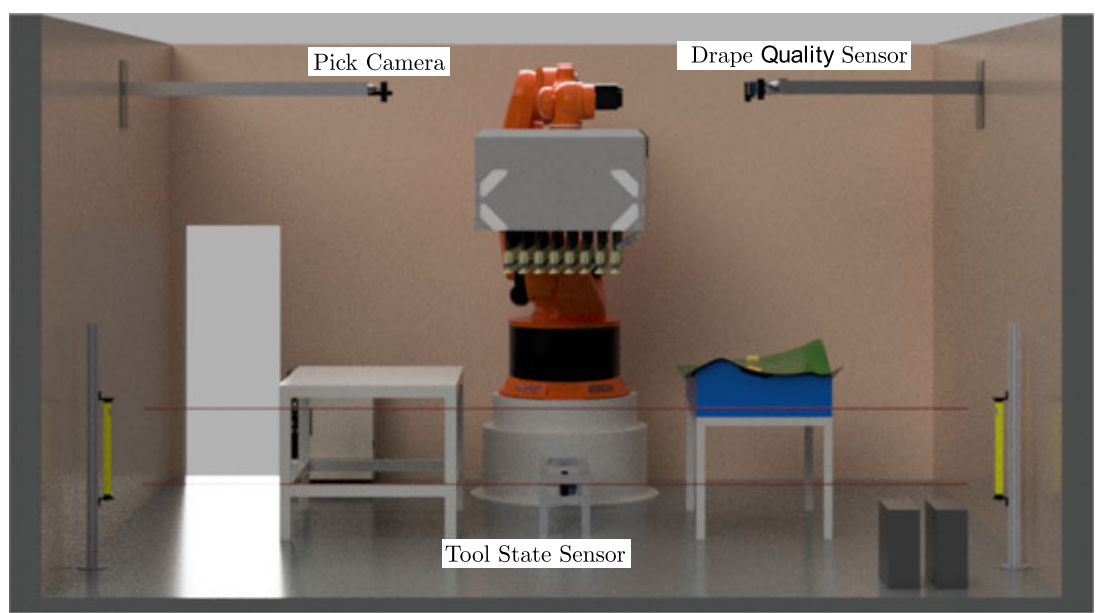

Fig. 7. Placement of sensors in the FlexDraper cell.

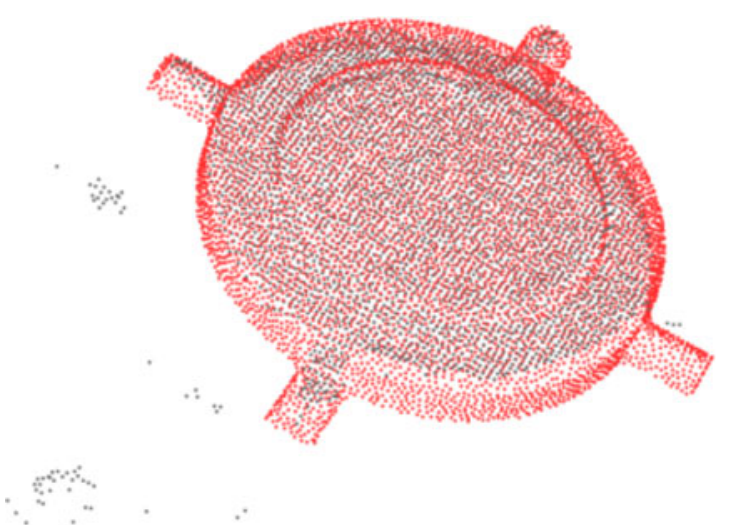

Fig. 8. Point cloud of one of the suction cups as captured by the tool state sensor.

The aforementioned tool state sensor serves two processes highlighted in Fig. 2:

- Estimate poses of suction cups

- Estimate preshape

The estimation of poses of suction cups is based on the measurements taken prior to picking the ply and it is necessary because due to the underactuation, the actual pose of each suction cup is unknown and impossible to model when no ply is attached. The estimated poses of suction cups are then used for predicting the locations, where the individual suction cups should pick up the ply.

For estimating the poses of suction cups, the 3D input data from the tool state sensor are acquired by scanning the suction cup array from below. The poses of the suction cups are recovered from the data by means of $3 \mathrm{D}$ pose estimation.

First, the 3D data are segmented into disjoint cells, corresponding to the individual suction cups, by means of a regular quadrilateral grid in camera space. Since the segmented regions remain constant, they are predefined in a manual operation. The pose of each suction cup is then recovered by robust alignment of the 3D CAD drawing of the suction cups.

Suction cups are found in the 3D data by fitting planes with the Random Sampling Consensus heuristic. The resulting locations and orientations are used for initializing point-to-plane-based Iterative closest point registration of the CAD suction cup to the data. Figure 8 shows alignment of a single suction cup, and Fig. 9 shows the resulting estimates of positions and orientations of the cups. 


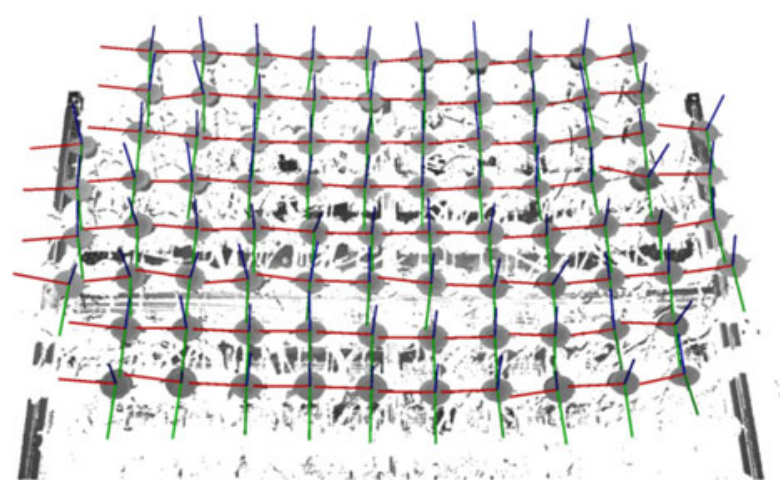

Fig. 9. Computed pose estimates of the suction cups.

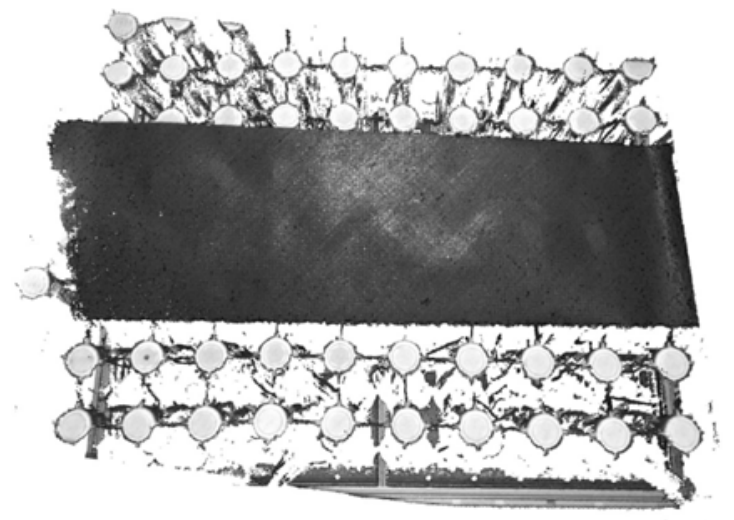

Fig. 10. Point cloud of a scanned fiber ply.

Estimate preshape is referring to the estimation of states of drape tool and ply after a ply is picked up. It is done based on the 3D scan of the ply, while it is held with the drape tool, combined with the states of drape tool and ply, which are predicted by the presented mathematical models.

The 3D data of the ply used in FlexDraper are acquired both from the tool state sensor and later on the drape quality sensor. Both sensors are structured light systems based on active stereo fringe projection, ${ }^{25}$ which is able to achieve very high accuracy and precision while keeping scan times in the order of a few seconds. ${ }^{26}$ The spatial encoding uses two frequency phase shifting with phase unwrapping based on the heterodyne principle. ${ }^{27}$ This encoding method is particularly flexible and allows for the detection and elimination of falsely encoded surface points, which will inevitably occur due to the complex optical properties of carbon fiber composites. ${ }^{28}$ Our experimentation shows that with careful consideration of these effects, excellent results of the 3D ply shapes can be achieved. Our solution is implemented with the tool state sensor, and Fig. 10 shows an example of a scanned ply.

The tool state sensor is comprised of two Gigabit Ethernet cameras (FLIR BFLY-PGE-23S6MC) and a LightCrafter 4500 programmable pattern projector configured through USB. The projector emits blue light $(465 \mathrm{~nm})$. Hardware triggers the cameras for synchronized display and captures the structured light pattern sequence. In order to reduce ambient lighting effects, the cameras are equipped with narrow band-pass filters matching the illumination wavelength.

\section{Proof-of-Concept Experimental Results}

Most of the experiments performed until now on the experimental physical model of the system have had the aim to test and verify the electrical and mechanical robustness of the proposed solution to the integrated system. Initially, the pick and drape tests were taught manually and performed for making qualitative checks of system's repeatability, indicating that it can be modeled deterministically. The tests showed a position variation of the ply post drape of only a few millimeters, which is a promising result, since the tool state sensor is not yet used for correcting the positions. Moreover, the purpose 

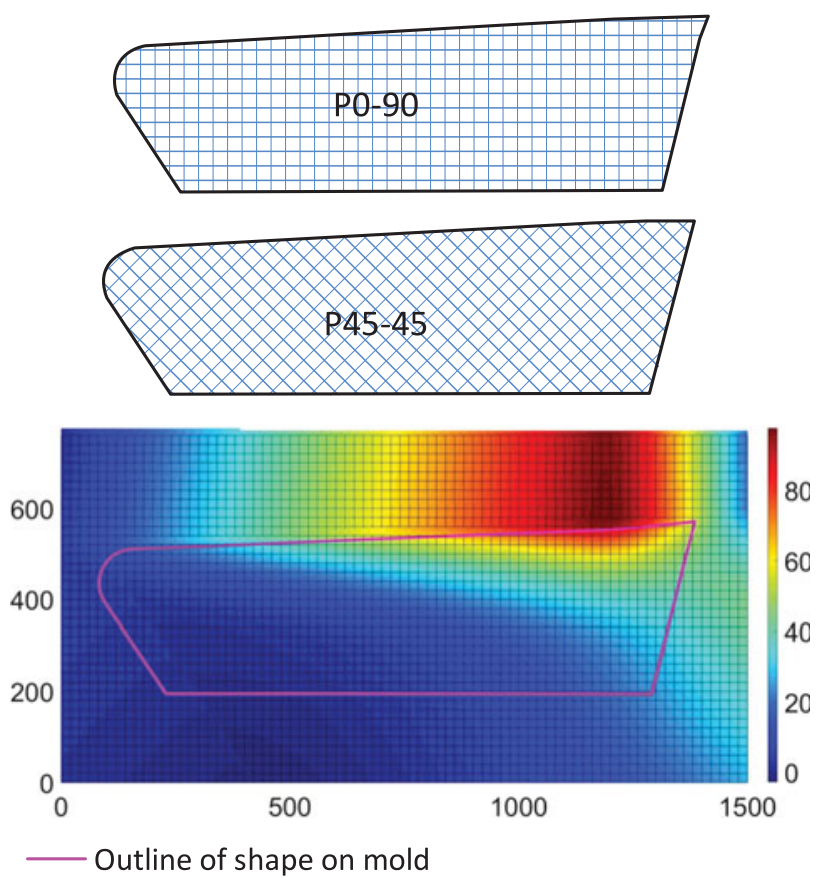

Fig. 11. Experimental set-up: two different orientations of prepreg material and a double curved mold. The dimensions of the mold are in $\mathrm{mm}$ and the height is color coded as shown on the scale bar.

of these manually taught tests has been to study if the system would be able to produce the required draping quality.

The conventional lay-up table of the part consists of a multilayer prepreg material with both full plies, covering the complete part surface, and partial plies, acting as local reinforcement layers in the assembly areas. The plies used in the Proof-of-Concept experiments are full pies, as shown in Fig. 11. They were draped on the mold with one layer of prepreg material, placed manually on the mold prior to the experiments and with no draping quality issues. A typical edge panel of $1200 \times 350 \mathrm{~mm}$ with relatively low curvature has been selected. The chosen part has the shape and complexity representing a large product family of similar aerospace parts. The advantage of using this part over a more complex or larger and more material intensive part is also the possibility for quickly testing the different aspects of automation during the development of the automated process. The prepreg material used in the experiments had the 4 harness-satin weave fiber pattern. Two different orientations of the prepreg material with respect to the mold were tested: $P 0-90$ and $P 45-45$. The orientation $P 0-90$ has the fibers aligned to the edges of the mold and the orientation $P 45-45$ has the fibers rotated $45^{\circ}$ with respect to the edges of the mold.

In the performed experiments with automated programming, the gripper grid preshaped the ply, in order to approximate the shape of the mold, and positioned the robot $20 \mathrm{~mm}$ above the drape location. Starting in one corner, the linear actuators of the tool created a wave-like motion, draping the ply onto the mold. Two types of wave strategies were tested: square wave strategy and skewed wave strategy, both shown in Fig. 12. The square wave strategy is staring from any single cup and moves outward in a ring-like wave. The skewed wave strategy is staring in a corner and moves on a straight line with the constant angle. Both strategies were applied to two different ply orientations $P 0-90$ and $P 45-45$, shown in Fig. 11.

The experimental results showed that the square wave strategy applied to $P 0-90$ gives little to no wrinkling of the material. Whereas applying the same strategy to $P 45-45$ resulted in significantly more wrinkling, which was caused by forces stretching the material in the diagonal direction of the fibers. Changing the draping strategy to the skewed wave reduced wrinkling of $P 45-45$ samples. For validation purpose, the skewed strategy was also applied to $P 0-90$, and as expected, it increased wrinkling in the drape quality.

A Proof-of-Concept experiment is demonstrated on the experimental physical model of the system, shown in Fig. 13. The results of an automated draping process can be seen in Fig. 14. Figure 14a 

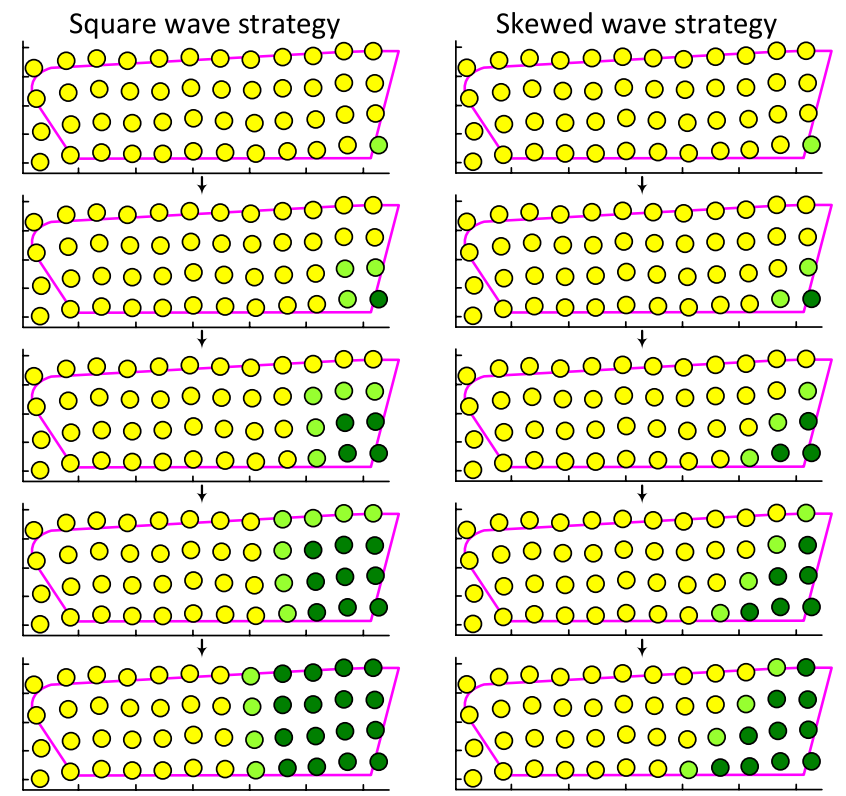

Fig. 12. Drape strategies used in the experiments, where color coding indicates position of the suction cup. Yellow is the preshape position, light green is the middle position and dark green is contact with the mold. The purple line illustrates the boundary of the ply.

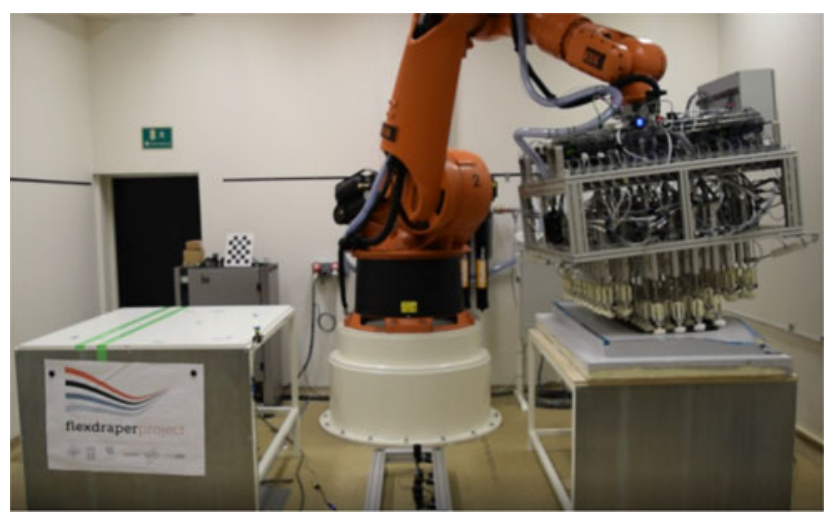

Fig. 13. (taken from Supplementary Video 1. Please refer to the supplementary material for the full video): Proof-of-Concept experiment showing automated draping process, performed on the experimental physical model of the system.

(a)

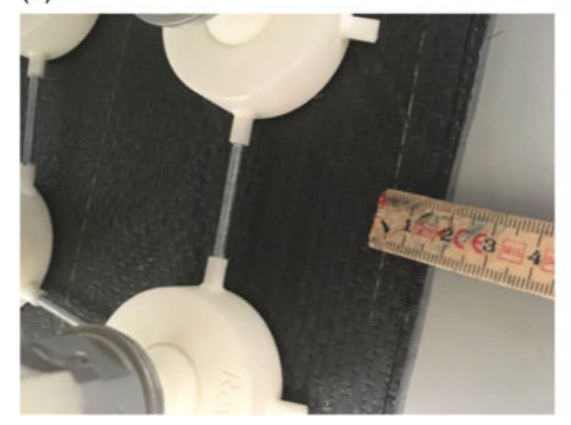

(b)

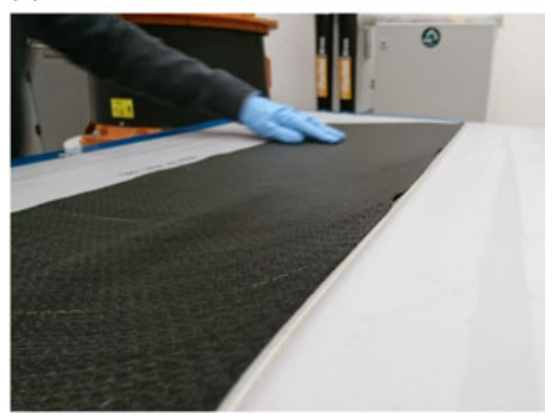

Fig. 14. Results of draping a $1200 \times 350 \mathrm{~mm}$ ply onto a double curved low curvature surface. (a) After the automatic drape the outline of the ply is offset approximately $5 \mathrm{~mm}$. (b) Draping result with only minor air intrusions, which are fixed during vacuum debulk.

shows the alignment of the ply. Its offset is approximately $5 \mathrm{~mm}$. Figure $14 \mathrm{~b}$ shows the case example of the overall draping result. It contains minor air intrusions, which can be handled by the sub-sequential vacuum debulking process. 


\section{Conclusion and Future Work}

The main contribution of this paper is the novel design and implementation of a new system for automatic robotic draping of prepreg fiber fabrics onto double curved molds. The various hardware and software components have been described in detail, and the integration issue of these components to the complete solution has been discussed. Finally, Proof-of-Concept experiments are conducted on the experimental physical model of the system, and the results are presented.

The system has been tested on an actual, double curved part from an aircraft. The prepreg material used in the experiments had the 4 harness-satin weave pattern, and it was draped onto the mold using two different orientations: $P 0-90$ and $P 45-45$. For both orientations, two draping strategies were applied: square weave strategy and skewed wave strategy. The final drape was then inspected for quality, represented by the number of wrinkles and air intrusions. Based on the Proof-Of-Concept experimental results, it can be concluded that the draping strategies have to be adapted to the specific drapes, as the shape of the part and the orientation of fibers in the ply with regard to the mold have an impact on the resulting draping quality. This underlines also the importance of having realistic ply and tool models available for offline planning of suited drape strategies.

Various technologies still need to be developed before the system is ready for production. The current drape tool is a part of the experimental physical model of the system with many 3D printed parts that will be replaced. Moreover, it is desired to simplify the layout of the drape tool, further reduce the weight and study other kinematic structures. In addition, an open/close mechanism for the suction will be installed for each suction cup. Furthermore, the development of the tool state sensor will be finalized, and the two additional sensors, which are the Pick Camera and the drape quality sensor, will be installed and tested.

The models will be improved based on the inputs from the experimental tests with the tool state sensor and by direct tests of the material properties. Furthermore, the current version of applying the wave-like draping strategy is very premature and will be substantially improved and integrated with a learning strategy based on the feedback from the drape quality sensor.

Even though the system is still at an early stage of development, the initial trials have been positive. We therefore convinced that the FlexDraper system as presented constitutes a feasible approach toward fully automated draping of prepreg composite fabrics.

\section{Acknowledgment}

The authors would like to thank the Innovation Fund Denmark for their financial support to the FlexDraper project. The financial support from Manufacturing Academy of Denmark and Lockheed Martin is also gratefully acknowledged.

\section{Supplementary Material}

To view supplementary material for this article, please visit https://doi.org/10.1017/ S0263574720000193.

\section{References}

1. D. H. J. Lukaszewicz, C. Ward and K. D. Potter, "The engineering aspects of automated prepreg layup: History, present and future," Composites Part B 43(3), 997-1009 (2012). DOI: 10.1016/j.compositesb.2011.12.003.

2. R. Molfino, M. Zoppi, F. Cepolina, J. Yousef and E. E. Cepolina, "Design of a Hyper-Flexible Cell for Handling 3D Carbon Fiber Fabric," Proceedings of the 2014 International Conference on Theoretical. Mechanics and Applied Mechanics (TMAM'14). Venice, Italy, pp. 165-170. https://pdfs.semanticscholar. org/da24/dbc48c622c8037b3188229bed051bf1734f7.pdf.

3. A. Björnsson and K. Johansen, "Automated material handling in composite manufacturing using pick-and-place systems review," Robotics Comput. Integr. Manuf. 51, 222-229 (2018). DOI: 10.1016/j.rcim.2017.12.003.

4. M. T. Kordi, M. Husing and B. Corves, "Development of a Multifunctional Robot End-Effector System for Automated Manufacture of Textile Preforms," Proceedings of the 2007 International Conference on Advanced Intelligent Mechatronic.

5. T. Gerngross and D. Nieberl, "Automated manufacturing of large, three-dimensional CFRP parts from dry textiles," CEAS Aeronaut J. 7, 241-257 (2016). DOI: 10.1007/s13272-016-0184-5.

6. F. Förster, F. Ballier, S. Coutandin, A. Defranceski and J. Fleischer, "Manufacturing of Textile Preforms with an Intelligent Draping and Gripping System," Proceedings of the 1st Cirp Conference on Composite Materials Parts Manufacturing (Elsevier) 66, 39-44 (2017). DOI: 10.1016/j.procir.2017.03.370. 
7. G. Gardiner, "Automated preforming: Intelligent automation in pick-and-place systems," CompositesWorld (2017); 10/22.

8. G. Gardiner, "Improving one-piece aerostructures by automating preforming," CompositesWorld (2017); $1 / 15$.

9. A. Angerer, C. Ehinger, A. Hoffmann, W. Reif and G. Reinhart, "Design of an Automation System for Preforming Processes in Aerospace Industries," Proceedings of the 2011 IEEE International Conference on Automation Science and Engineering (IEEE). DOI: 10.1109/CASE.2011.6042411.

10. C. Ehinger and G. Reinhart, "Robot-based automation system for the flexible preforming of single-layer cut-outs in composite industry," Product. Eng. Res. Dev. 8, 559-565 (2014). DOI: 10.1007/s11740-014-0546-y.

11. S. Sterk, "Low Cost Automated Manufacturing of an A320 Slat Fairing," Proceedings of SAMPE Europe SEICO 2014 - 35th International Technical Conference \& Forum, Paris, France.

12. COALESCE. Cost efficient advanced leading edge structure (2009). http://www.structures.ethz.ch/ research/computational-structural-mechanics/COALESCE2.html.

13. K. Campbell, "Tsb Affordable Composites Manufacturing - Grand Challenge," Presentation at ACCIS Annual Conference (2012). http://www.axillium.com/i-composites/sites/default/files/i-Composites\%20$\% 20$ Picking\%20and\%20Placing\%20of\%20Dry\%20Fibre.pdf.

14. M. Quigley, B. Gerkey, K. Conley, J. Faust, T. Foote, J. Leibs, E. Berger, R. Wheeler and A. Ng, "Ros: An Open-Source Robot Operating System," Proceedings of the IEEE International Conference on Robotics and Automation (ICRA) Workshop on Open Source Robotics, Kobe, Japan (2009). https://pdfs.semantics cholar.org/d45e/aee8b2e047306329e5dbfc954e6dd318ca1e.pdf?_ga=2.240291008.2127394806.15870361 08-362228064.1578833333.

15. F. Van Der Ween, "Algorithms for draping fabrics on doubly-curved surfaces," Int. J. Numer. Methods Eng. 31(7), 1415-1426 (1991).

16. T. Liu, A. Bargteil, J. O'Brien and L. Kavan, "Fast simulation of mass-spring systems," ACM Trans. Graphics 32(6), Article 214 (2013). DOI: 10.1145/2508363.2508406.

17. O. W. Nielsen, C. Schlette and H. G. Petersen, "Fast and Simple Model for Free Hanging, Pre-Impregnated Carbon Fibre Material," Icinco 2018 (2018) pp. 7-14.

18. T. Gereke, O. Döbrich, M. Hübner and C. Cherif, "Experimental and computational composite textile reinforcement forming: A review," Compos. Part A Appl. Sci. Manuf. 46(1), 1-10 (2013). DOI: 10.1016/j.compositesa.2012.10.004.

19. J. Cao, R. Akkerman, P. Boisse, J. Chen, H. S. Cheng, E. F. de Graaf, J. L. Gorczyca, P. Harrison, G. Hivet, J. Launay, W. Lee, L. Liu, S. V. Lomov, A. Long, E. de Luycker, F. Morestin, J. Padvoikis, X. Q. Peng, J. Sherwood, T. Z. Stoilova, X. M. Tao, I. Verpoest, A. Willems, J. Wiggers, T. X. Yu and B. Zhu, "Characterization of mechanical behavior of woven fabrics: experimental methods and benchmark results," Compos. Part A Appl. Sci. Manuf. 39(6), 1037-1053 (2008). DOI: 10.1016/j.compositesa.2008.02.016.

20. C. Krogh, J. A. Glud and J. Jakobsen, "Modeling the robotic manipulation of woven carbon fiber prepreg plies onto double curved molds: A path dependent problem," J. Compos. Materials 53(15), 2149-2164 (2019).

21. R. M. Murray, Z. Li and S. S. Sastry, A Mathematical Introduction to Robotic Manipulation. 1 ed. (CRC Press, 1994). ISBN 9780849379819.

22. E. Minguzzi, "Rayleigh's dissipation function at work," Eur. J. Phys. 36(3), 035014 (2015). doi: 10.1088/ 0143-0807/36/3/035014. 1409.4041.

23. J.E. Marsden and M. West, "Discrete mechanics and variational integrators," Acta Numerica 10, 357-514 (2001). DOI: 10.1017/S096249290100006X.

24. O. A. Bauchau and A. Laulusa, "Review of contemporary approaches for constraint enforcement in multibody systems," ASME J. Comput. Nonlinear Dyn. 3(1), 011005 (2008). DOI: 10.1115/1.2803258.

25. P. Kühmstedt, C. Munckelt, M. Heinze, C. Bräuer-Burchardt and G. Notni, "3D Shape Measurement with Phase Correlation Based Fringe Projection," Proceedings of SPIE 6616. DOI: 10.1117/12.726119.

26. E. R. Eiríksson, J. Wilm, D. B. Pedersen and H. Aanæs, "Precision and Accuracy Parameters in Structured Light 3-d Scanning," International Archives of the Photogrammetry, Remote Sensing and Spatial Information Sciences, vol. XL-5/W8 (2016) pp. 7-15. DOI: 10.5194/isprs-archives-XL-5-W8-7-2016.

27. C. Reich, R. Ritter and J. Thesing, "White Light Heterodyne Principle for 3D-measurement," SPIE 3100, Sensors, Sensor Systems, and Sensor Data Processing, vol. 3100 (1997) pp. 236-244.

28. W. Palfinger, S. Thumfart and C. Eitzinger, "Photometric Stereo on Carbon Fiber Surfaces," 35th Workshop of the Austrian Association for Pattern Recognition. 